\title{
EDITORIAL
}

\section{Fighting child mortality}

\author{
Pneumococcal vaccines and rotavirus vaccines will help to reach the goal, set by the United \\ Nations Millennium Project, of a two-thirds decrease in child mortality by 2015.
}

As described previously on this page, pneumonia is one of the most important causes of child mortality ${ }^{1}$, closely followed by diarrhoea. A report on the progress towards the goals of the United Nations Millennium Project ${ }^{2}$, released in 2010, shows that these two illnesses account for nearly $30 \%$ of all deaths of children under 5 years old, a much higher percentage than that for the much more widely publicized diseases HIV, malaria and tuberculosis. The goal to decrease the child mortality rate by twothirds between 1990 and 2015 is laudable, but to reach this target, tools to fight the most common causes of child mortality are required. The good news is that several recent reports now show that such tools, in the form of new vaccines, are effective and ready to be implemented in the areas where they are most needed.

One-third of the 1.3 million diarrhoea-related deaths each year are due to rotavirus infection. In 2006, the WHO approved the use of two rotavirus vaccines, Rotarix (produced by GlaxoSmithKline) and RotaTeq (produced by Merck). Now, the results of post-implementation surveillance from several high- and middle-income countries, published recently in a supplement to the Pediatric Infectious Disease Journal (http://journals.lww.com/pidj/ toc/2011/01001), show that these vaccines have greatly decreased the number of rotavirus cases. The effects of the vaccines ranged from an $89-94 \%$ reduction in hospitalizations due to rotavirus infections in Queensland, Australia, to a $40 \%$ decline in diarrhoea-related hospitalizations in Mexico, whereas El Salvador saw a 69-81\% reduction in the rate of rotavirus-related hospitalizations. In addition, the effect of these vaccines extended beyond the targeted group of young children, as the rate of rotavirus infections among older, non-vaccinated children decreased, indicating wider protection in the community owing to the decreased levels of circulating virus.

Vaccination against pneumonia is likely to have a similar effect in the coming years. An estimated 2 million children under 5 years of age are killed by this disease annually, and more than 800,000 of these deaths are due to infection with Streptococcus pneumoniae. Two new pneumococcal vaccines, Prevenar 13 (produced by Pfizer) and Synflorix (produced by GlaxoSmithKline), were only recently introduced in developing nations to cover a broad spectrum of $S$. pneumoniae strains. But Prevenar 13 has already become part of the routine immunization programme in Nicaragua, and Kenya has also adopted these new pneumococcal vaccines; they will be rolled out in Sierra Leone, Yemen, Honduras and Guyana later this year, and then in other countries in Africa and the Middle East. What has allowed these vaccines to be introduced so rapidly in developing nations after their introduction in developed nations is the Advance Market Commitment (AMC) programme of the Global Alliance for Vaccines and Immunisation (GAVI; www.gavialliance.org/), which provides incentives for the vaccine manufacturers to develop vaccines and distribute them in developing countries.

Wider implementation of the rotavirus vaccines and the pneumococcal vaccines is likely to save millions of lives - the pneumococcal vaccines alone are expected to save 700,000 lives by 2015 and more than 7 million by 2030. However, this will require a continued financial commitment from developed and developing nations, and philanthropic organizations, as well as the manufacturers, who make the pneumococcal vaccines available for a fraction above the price of production. The vaccines remain expensive (about US\$5 per dose), but in developing nations each life saved saves around $\$ 3,000$, supporting the case for investing in the wide distribution of the vaccines.

At a conference that will be hosted by the UK government in June 2011 and will include many world leaders, GAVI will make the case for additional funding; it estimates that it needs an additional $\$ 3.7$ billion over the next 5 years to bring these and other vaccines to developing nations.

At the current rate of decline in child mortality, the goal of the Millennium Project will not be achieved. However, the rate of decline has been increasing, and the rotavirus vaccines and pneumococcal vaccines will increase this rate even further. With better vaccines available, we run out of excuses for allowing children to die of preventable diseases. As stated in the introduction to the Pediatric Infectious Disease Journal supplement: "The time to introduce these lifesaving interventions is now"3.

1. No authors listed. Closing the GAPP on pneumonia. Nature Rev. Microbiol. 7, 838 (2009).

2. United Nations. The Millennium Development Goals report 2010 United Nations [online], http://www.un.org/millenniumgoals/pdf/MDG Report 2010 En r15 -low res 20100615 - pdf (2010)

than 7 million by

2030.
Patel, M. M. et al. Real-world impact of rotavirus vaccination. Pediatr. Infect. Dis. J. 30, S1-S5 (2011). 